J. Clin. Chem. Clin. Biochem.

Vol. 15,1977 , pp. $515-518$

\title{
Dünnschichtchromatographische Methode zum Screening auf 4-Hydroxy-3-methoxy-mandelsäure im Harn ohne Extraktion
}

\author{
Von R. Knuppen .
}

Abteilung für Biochemische Endokrinologie der Medizinischen Hochschule Lübeck,

R. Helger, F. Kraffczyk und H. Lang,

Biochemische Forschung, E. Merck Darmstadt

Eingegangen am 6. Januar/20. Mai 1977)

Zusammenfassung: Es wird ein Test (DC-Test) beschrieben, mit dem 4-Hydroxy-3-methoxymandelsäure (HMMS) im Urin dünnschichtchromatographisch halbquantitativ bestimmt werden kann. Ein der Tagesausscheidung aliquotes Harnvolumen wird ohne Extraktion und Konzentration auf einer speziellen Dünnschichtfertigplatte eindimensional chromatographiert und die HMMS anhand von mitgeführten Standards halbquantitativ bestimmt.

Bei 102 Hochdruck-Patienten im Alter über 18 Jahre wurde die HMMS-Ausscheidung im 24-Stunden-Harn parallel mit der Methode von Pisano (Pisano, J. J., Crout, J. R. \& Abraham, D. (1962), Clin. Chim. Acta 7, 285-291) und dem DC-Test untersucht. Ubereinstimmende Ergebnisse mit beiden Methoden werden in 99 Fällen erhalten. Bei den restlichen 3 Fällen, in welchen die Ergebnisse der Methode von Pisano im pathologischen Bereich und die Ergebnisse des DC-Tèsts im Normalbereich bzw. im Warnbereich liegen, handelt es sich wahrscheinlich um falschpositive Ergebnisse der Methode von Pisano.

Der DC-Test wird als Screening-Methode empfohlen.

Thin layer chromatography screening test for semiquantitative detection of 4-hydroxy-3-methoxy mandelic acid (HMMA) in urine without extraction

Summary: A thin layer chromatography (TLC) screening test is described, which allows the semiquantitative determination of HMMA in urine samples. From the collected 24 hour urine a measured sample is applied without extraction to a special TLC plate, which is developed in one dimension. In comparison to a series of graded standard solutions the daily HMMA output can be estimated semiquantitatively.

In 102 patients over 18 years of age, suffering from high blood pressure, the daily HMMA output was estimated by both the Pisano method (Pisano, J. J., Crout, J. R. \& Abraham, D. (1962), Clin. Chim. Acta 7, 285-291) and the TLC method. Coincident results with both methods were obtained in 99 of these cases. The residual 3 cases show pathological results with the Pisano method and results in the normal range or the warning range, respectively, with the TLC method. These cases can probably be classified as false positive results of the Pisano method.

The TLC method is recommended as a screening test for estimating the daily HMMA excretion.

\section{Ëinfuihrung}

Für die Diagnostik catecholaminproduzierender Tumoren hat die Bestimmung der Catecholamine und der 4-Hydroxý-3-methoxy-mandelsäure (HMMS) im Urin eine große Bedeutung erlangt. Besonders die quantitative Erfassung der HMMS, die im Urin des Menschen in Milligramm-Mengen ausgeschieden wird und das quantitativ wichtigste Abbauprodukt der Catecholamine Noradrenalin und Adrenalin darstellt, ist von erheblichem diagnostischen Wert. In klinisch-chemischen Laboratorien erfolgt die Bestimmung der HMMS im Urin meist nach der von Pisano et al (1) beschriebenen Methode. Bei diesem Verfahren wird die aus dem Urin mittels organischer Lösungsmittel extrahierte HMMS mit Natrium-meta-perjodat zu Vanillin (4-Hydroxy-3-methoxy-benzaldehyd) oxidiert und anschließend die Absorption des Vanillins in einer wäßrigen Kaliumcarbonatlösung bei $360 \mathrm{~nm}$ gemessen. $\mathrm{Da}$ 
gelegentlich das Absorptionsspektrum des Vanillins entweder durch Eigenabsorption von mitgeschleppten Fremdstoffen überlagert werden kann oder hypsochrom verschoben wird, bevorzugen manche Untersucher zur Erhöhung der Spezifität die gaschromatographische Endpunktsbestimmung des Vanillins. Nach einem von Badella et al (2) angegebenen Verfahren wird der Harnextrakt dünnschichtchromatographisch aufgetrennt und die HMMS nach Anfärbung mit diazotiertem 4-Nitroanilin visuell oder densitometrisch bestimmt.

Im Hinblick darauf, daß einerseits den meisten klinischchemischen Laboratorien die Bestimmung der HMMS nach den Verfahren von Pisano oder Badella, wobei mehrfach Extraktionsschritte erforderlich sind, zu arbeitsaufwendig erscheint, andererseits jedoch die Erfassung der HMMS zur Diagnostik catecholaminproduzierender Tumoren von großem klinischen Interesse ist, wurden in den letzten Jahren zahlreiche Versuche unternommen, diese Phenolcarbonsäure im Urin des Menschen ohne die Notwendigkeit aufwendiger Extraktionsschritte quantitativ zu erfassen. Von Dittmann (3) wurde eine Methode angegeben, bei der Harn direkt auf eine Dünnschichtplatte aufgetragen wird; wegen der Störungen durch Salze und Harnstoff sowie wegen der unvollständigen Auftrennung verschiedener Metabolite erwies sich jedoch dieses Verfahren als unbefriedigend. Vor einigen Jahren berichteten Kraffczyk \& Helger (4) über ein dünnschichtchromatographisches Trennsystem, das es gestattet, HMMS halbquantitativ zu bestimmen.

Bei diesem Verfahren wird durch die Verwendung einer stationären Phase auf Cellulosebasis mit Anionenaustauscher-Eigenschaften und eines sauren Elutionsmittels eine direkte Chromatographie von Harn auf Dünnschichtplaten ermöglicht; die Trennleistung dieses Systems wird durch Salze und Harnstoff nicht beeinflußt. Im Vergleich zu den bisher bekannten dünnschichtchromatographischen Methoden mit gleichen Trennleistungen zeichnet sich die beschriebene Methode durch den Verzicht auf Extraktionsschritte und Konzentrierung der Extrakte aus.

In der vorliegenden Arbeit wurde unter den Bedingungen der klinischen Routineanalytik die Ausscheidung der HMMS in 24-Stunden-Urinen mit Hilfe des Verfahrens von Kraffczyk \& Helger erfaßt und zum Vergleich den mit der Methode von Pisano ermittelten Werten gegenübergestellt. Insbesondere wurde die Frage geprüft, ob der Untersucher in der Lage ist, aufgrund des beschriebenen Screeningtests die Ausscheidung der HMMS hinreichend zuverlässig als normal einzustufen und die Anwendung der arbeitsaufwendigen photometrischen Methoden auf die wenigen Fälle mit erhöhter Ausscheidung zu beschränken.

\section{Material und Methoden}

Zur Analyse kamen 24-Stunden-Harne von Hypertonikern; alle Urine wurden über $30 \mathrm{ml} 6 \mathrm{~mol} / 1 \mathrm{HCl}$ in Plastikflaschen gesammelt. Harnproben, in denen der pH-Wert oberhalb $3 \mathrm{lag}$, wur- den verworfen. Medikamente, insbesondere Antisympathotonika, wurden 6 Tage vor der Sammelperiode abgesetzt. Die Urine wurden entweder sofort am Einsendetag analysiert oder bei $-20^{\circ} \mathrm{C}$ tiefgefroren für höchstens 8 Tage aufbewahrt.

Quantitative Bestimmung von 4-Hydroxy-3-methoxymandelsäure

Die quantitative Bestimmung der 4-Hydroxy-3-methoxy-mandelsäure erfolgte in Duplikaten nach der Methode von Pisano (1) durch Aufnahme der Absorptionsspektren von Vanillin im Bereich von 320-400 nm mit einem registrierenden Spektralphotometer (Shimadzu UV 200). Batho- oder hypsochrom verschobene Spektren wurden nicht ausgewertet.

Screeningtest auf 4-Hydroxy-3-methoxy-mandelsäure ${ }^{1}$ ) Materialien

$2 \mu \mathrm{l}$-Kapillaren und speziell präparierte DC-Fertigplatten $(20 \times 10 \mathrm{~cm})$.

\section{Reagenzien und Lösungsmittel}

A) Tris-Tabletten (240 mg Tris (hydroxymethyl)-aminomethan, $60 \mathrm{mg} \mathrm{NaHCO}{ }_{3}$ und $30 \mathrm{mg} \mathrm{Li}_{2} \mathrm{CO}_{3}$ ),

B) Chloroform/Butanol-(1)/Ethanol (Volumina, $5 \mathrm{ml}+55 \mathrm{ml}$ $+10 \mathrm{ml}$ ),

C) Essigsäure/Wasser (Volumina, $100 \mathrm{ml}+100 \mathrm{ml}$ ),

D) 4-Nitroanilinlösung (11 mmol/1 4-Nitroanilin und $0.55 \mathrm{~mol} / \mathrm{l}$ $\mathrm{HCl}$ ),

E) Natriumnitrit-Lösung ( $900 \mathrm{mmol} / 1 \mathrm{NaNO}_{2}$ ),

F) Natriumcarbonat-Lösung ( $\left.1 \mathrm{~mol} / 1 \mathrm{Na}_{2} \mathrm{CO}_{3}\right)$ und

G-J) Standards St 4, St 6, St 8 und St $12(8,12,16$ bzw. $24 \mathrm{mg} / \mathrm{l}$ 4-Hydroxy-3-methoxy-mandelsäure, wo bei die Standards St 4 und St 6 nicht in der Testpackung vorhanden sind, sondern selbst angesetzt wurden.

\section{Herstellung der Lösungen}

Laufmittel

Zur Herstellung des Laufmittels Chloroform/Butanol-(1)/Ethanol/Essigsäure/Wasser (Volumina, $5 \mathrm{ml}+55 \mathrm{ml}+10 \mathrm{ml}+15 \mathrm{ml}$ $+15 \mathrm{ml}$ ) werden $28 \mathrm{ml}$ der Lösung B mit $12 \mathrm{ml}$ der Lösung C gemischt. Das gebrauchsfertige Laufmittel ist bei Raumtemperatur 12 Stunden haltbar.

\section{Nachweisreagenz}

Zu $6 \mathrm{ml}$ 4-Nitroanilin-Lösung (D) fügt man $0.1 \mathrm{ml}$ NatriumnitritLösung (E) und mischt. Dann fügt man $6 \mathrm{ml} \mathrm{Natriumcarbonat-}$ Lösung $(F)$ hinzu und mischt. Die nun gebrauchsfertige Lösung ist bei Raumtemperatur 30 min haltbar.

\section{Standardlösungen}

$\mathrm{Zu}$ den Standards $\mathrm{G}-\mathrm{J}$ fügt man je 1 ml dest. Wasser und löst. Die gebrauchsfertigen Lösungen sind bei 0 bis $+4{ }^{\circ} \mathrm{C}$ einen Monat haltbar.

\section{Ausführung des Screeningtests}

\section{Vorbereitung der Harnproben}

Das Volumen der 24-Stunden-Urine wurde bestimmt und 1/100 der Harnmenge in einen $50 \mathrm{ml}$ Meßzylinder gegeben. In der Harnprobe wurde eine Tris-Tablette (A) aufgelöst und danach soviel dest. Wasser hinzugegeben, daß jeweils volle $5,10,15$ bzw. $20 \mathrm{ml}$ usw. erreicht wurden.

\section{Auftragen der Proben und Standards}

An den auf Abbildung 1 bezeichneten Punkten der Dünnschichtplatte wurden je $2 \mu$ l der Standardlösungen St 4, St 6, St 8 und St 12 mit Hilfe der $2 \mu$-Kapillare punktförmig aufgetragen. Die Auftragsmenge der Harnprobe richtete sich nach dem Volu-

1) Reagenziensatz Merckotest: DC HMMS, Merck No. 3371 bzw. DC-Fertigplatte PEI-Cellulose, Merck No. 5722. 


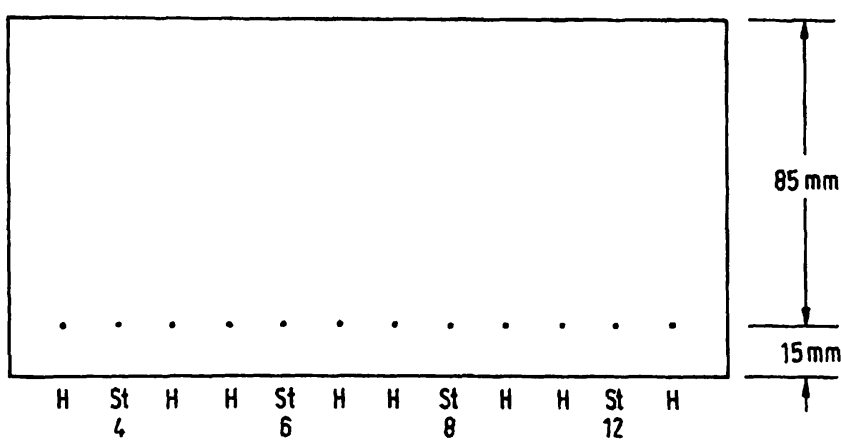

Ább. 1. Dünnschichtchromatographischer Screeningtest für 4-Hydroxy-3-methoxy-mandelsäure Auftrage-Schema für die DC-Platten

$\mathrm{H}=$ Harnproben

St $=$ Standardlösungen (von links nach rechts: St 4, St 6, St 8, St 12).

men der Analysenprobe; je $5 \mathrm{ml}$ Analysenprobe wurden $2 \mu \mathrm{l}$ auf einen Punkt aufgetragen, nach jedem Auftrag wurde kurz getrocknet.

\section{Entwicklung der Dünnschichtchromatogramme}

Es wurde zweifach mit dem Laufmittel entwickelt; die Dauer der Entwicklung betrug insgesamt etwa 3 Stunden. Nach der ersten Entwicklung wurde mit einem Heizlüfter, nach der zweiten Entwicklung zunächst mit dem Heizlüfter und dann 3 min bei $80^{\circ} \mathrm{C}$ im Trockenschrank getrocknet.

\section{Nachweis und Auswertung}

Nach dem Abdampfen der organischen Lösungsmittel wurde die DC-Platte mit dem Nachweisreagenz gleichmäßig besprüht und $3 \mathrm{~min}$ bei $80^{\circ} \mathrm{C}$ getrocknet. Danach wurde die Platte $30 \mathrm{~min}$ im Dunkeln bei Raumtemperatur liegengelassen. Zur Auswertung wurde die Platte auf einen Leuchtkasten gelegt und unabhängig von 3 Untersuchern Fleckengröße und Intensität der Analysenprobe mit den Standards verglichen. Es wurden nur solche Dünnschichtchromatogramme ausgewertet, die eine eindeutige Unterscheidung der Standardproben zuließen.

\section{Ergebnisse}

Bei Hochdruck-Patienten im Alter über 18 Jahre wurde im 24-Stunden-Harn 4-Hydroxy-3-methoxy-mandelsäure parallel mit der Methode von Pisano und der DC-Methode bestimmt. Tagesharne mit einem Volumen über $2000 \mathrm{ml}$ wurden von der Untersuchung ausgeschlossen. Die Ergebnisse von 105 Patienten wurden zur Auswertung herangezogen. Bei 45 dieser Patienten wurden die Bestimmungen doppelt oder dreifach an verschiedenen Tagesharnen durchgefuihrt, die an aufeinanderfolgenden Tagen unter möglichst identischen Bedingungen gewonnen worden waren. Die Ergebnisse der Mehrfachbestimmungen wurden.gemittelt und auf diese Weise in das Kollektiv einbezogen.

Aus diesem Kollektiv wurden nachträglich 3 Patienten ausgeschieden: 1 Patient unter Methyldopa-Medikation, 1 Patient unter hohen Dosen von Psychopharmaka und 1 Patient, in dessen Harn große Mengen nicht identifizierter Arzneimittel-Metaboliten nachgewiesen wurden.

Die Ergebnisse der Untersuchungen am verbleibenden Kollektiv von 102 Patienten sind in Abbildung 2 als
Tab. 1. Ausscheidung von 4-Hydroxy-3-methoxy-mandelsäure (HMMS) im 24 Stunden-Urin.

Vergleich der Ergebnisse der Methode von Pisano und der dünnschichtchromatographischen Methode $(n=102)$.

Methode

von Pisano: pathologisch ab $8 \mathrm{mg} / 24 \mathrm{~h}$ HMMS

DC-Methode: Warnbereich ab $6 \mathrm{mg} / 24 \mathrm{~h}$ HMMS pathologisch ab $8 \mathrm{mg} / 24 \mathrm{~h} \mathrm{HMMS}$

\begin{tabular}{|c|c|c|c|c|c|}
\hline \multirow{2}{*}{$\begin{array}{l}\text { Anzahl } \\
\text { Ergebnisse }\end{array}$} & \multicolumn{2}{|c|}{ Methode von Pisano } & \multicolumn{2}{|c|}{ DC-Methode } & \multirow[b]{2}{*}{$\begin{array}{l}\text { patho- } \\
\text { logisch }\end{array}$} \\
\hline & normal & $\begin{array}{l}\text { patho- } \\
\text { logisch }\end{array}$ & normal & $\begin{array}{l}\text { Warn- } \\
\text { bereich }\end{array}$ & \\
\hline 90 & + & & + & & \\
\hline $\begin{array}{l}7 \\
0\end{array}$ & $\begin{array}{l}+ \\
+\end{array}$ & & & + & + \\
\hline 2 & & + & + & & \\
\hline 1 & & + & & + & \\
\hline 2 & & + & & & + \\
\hline
\end{tabular}

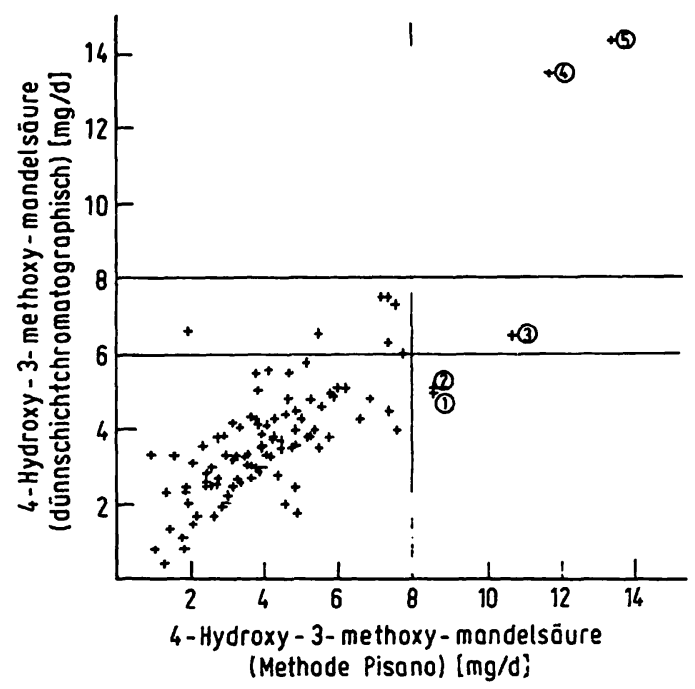

Abb. 2. Ausscheidung von 4-Hydroxy-3-methoxy-mandelsäure (HMMS) im 24 Stunden-Harn von Hochdruckkranken $\mathrm{n}=102$

$\mathrm{x}=$ Methode Pisano: Obergrenze Normal $=8 \mathrm{mg} / 24 \mathrm{~h}$

$y=$ DC-Methode: Warngrenze $\quad=6 \mathrm{mg} / 24 \mathrm{~h}$

HMMS

Obergrenze Normal $=8 \mathrm{mg} / 24 \mathrm{~h}$
HMMS

Bewertung der Ergebnisse siehe Text.

Korrelation der beiden Methoden dargestellt. Wenn für HMMS, bestimmt mit der Methode nach Pisano. eine Obergrenze der Norm von $8 \mathrm{mg} / 24 \mathrm{~h}$ gilt und für die DC-Methode $6 \mathrm{mg} / 24 \mathrm{~h}$ HMMS als Warngrenze sowie $8 \mathrm{mg} / 24 \mathrm{~h}$ HMMS als Obergrenze der Norm festgelegt werden, ergeben sich die in Tabelle 1 zusammengefaßten Zahlen: bei $95 \%$ der Patienten wurden mit beiden Methoden normale Ergebnisse gemessen, davon lagen bei 7\% die Ergebnisse der DC-Methode im Warnbereich. in dem die Bestimmung noch einmal wiederholt werden sollte. Bei keinem Patienten wurden nach der Methode von Pisano bei normalem Ergebnis pathologische Ergebnisse der DC-Methode erhalten. Von den 5 Patienten mit nach der Methode von Pisano pathologischem Er- 
gebnis hatten 2 ein normales DC-Ergebnis, 1 ein DC-Ergebnis im Warnbereich und 2 ein pathologisches DC-Ergebnis.

Bei den drei Patienten mit nach der Methode von Pisano pathologischem Ergebnis und Ergebnis der DC-Methode im Normal- bzw. Warnbereich wurden folgende Werte (Mittelwerte aus Dreifachbestimmungen) gemessen (Nummern $=$ Bezeichnung der Fälle in Abb. 2):

(1) HMMS Pisano $8,6 \mathrm{mg} / 24 \mathrm{~h}, \mathrm{HMMS}$ DC $5,5 \mathrm{mg} /$ $24 \mathrm{~h}$, Catecholamine $43 \mu \mathrm{g} / 24 \mathrm{~h}$;

(2) HMMS Pisano $8,6 \mathrm{mg} / 24 \mathrm{~h}, \mathrm{HMMS}$ DC $5,6 \mathrm{mg} /$ $24 \mathrm{~h}$, Catecholamine $58 \mu \mathrm{g} / 24 \mathrm{~h}$;

(3) HMMS Pisano $10,7 \mathrm{mg} / 24 \mathrm{~h}$, HMMS DC $6,5 \mathrm{mg} /$ $24 \mathrm{~h}$, Catecholamine $59 \mu \mathrm{g} / 24 \mathrm{~h}$.

Da die Bestimmung der Catecholamin-Ausscheidung in allen drei Fällen Werte im Normalbereich ergab, besteht die Möglichkeit, daß es sich um falsch positive Ergebnisse der Methode von Pisano handelt.

Die beiden Patienten mit pathologischen Ergebnissen sowohl der Pisano- als auch der DC-Methode zeigen deutlich pathologische Werte der Catecholamin-Ausscheidung:

(4) $159 \mu \mathrm{g} / 24 \mathrm{~h}$,
(5) $427 \mu \mathrm{g} / 24 \mathrm{~h}$.

\section{Diskussion}

Ein Vergleich der beiden zur Bestimmung der HMMS verwendeten Methoden soll ihre Vor- und Nachteile aufzeigen.

Die Methode von Pisano, die eine exakte quantitative Bestimmung der HMMS im Urin gestattet, beinhaltet mehrere Extraktionsschritte und ist damit zeitraubend und relativ aufwendig. Die photometrische Messung ist einfach und exakt; es kann jedoch nicht erkannt werden, ob Verluste bei der Aufarbeitung und Störungen bei der Oxidation zu Vanillin auftreten oder Absorptionen durch die Anwesenheit von Arzneimitteln bzw. deren Metaboliten vorliegen. Solche Störungen können zwar durch den
Einsatz radioaktiver HMMS und durch zusätzliche chromatographische Methoden erkannt und ausgeschaltet werden, doch dürften diese zusätzlichen Schritte in der Routine keine breite Anwendung finden.

Bei der DC-Methode fallen die Extraktions- und Konzentrationsschritte weg, so daß Aufarbeitungsverluste nicht auftreten. Interferenżen durch Arzneimittel und deren Metaboliten können wegen der unterschiedlichen $R_{F}$ Werte und der differenzierten Färbung bei der Diazotierung erkannt werden. Wie durch Versuche an Probanden nachgewiesen werden konnte, bewirken auch Bananen und ähnliche für die Methode von Pisano beschriebene Störfaktoren aus der Nahrung im DC-Test keine Störung. Auch in Urinen von schwangeren Frauen ließ sich die Ausscheidung der HMMS mit der DC-Methode einwandfrei bestimmen.

Die Durchführung des DC-Testes sollte jedoch nur durch in der Dünnschichtchromatographie geübte Fachkräfte erfolgen; dies betrifft insbesondere das Besprühen der Platten und die Auswertung der Flecken. Es sollte geprüft werden, ob eine quantitative Auswertung der DC-Platten, zum Beispiel mit der Methode nach Maruna (5), sinnvoll ist. Wegen der relativen Unempfindlichkeit der DC-Methode bei HMMS-Werten, die unterhalb einer Ausscheidung von $4 \mathrm{mg} / 24 \mathrm{~h}$ liegen, ist der Test bei Kleinkindern nicht geeignet. Wenn das Volumen der zu analysierenden Harne mehr als $2000 \mathrm{ml}$ beträgt, bereitet die Auswertung der Chromatogramme öfters Schwierigkeiten. Hierbei wurde die Ausbildung von Doppelflecken in der Position der HMMS beobachtet; letzteres ist wahrscheinlich bedingt durch eine Bildung von zwei Startpositionen beim Auftragen relativ großer Harnmengen.

Die DC-Methode kann als Screeningtest empfohlen werden. Da die Werte für die HMMS-Ausscheidung bei catecholaminproduzierenden Tumoren in der Mehrzahl der Fälle oberhalb $10 \mathrm{mg} / 24 \mathrm{~h}$ liegen (6) (10\% der Fälle unter $10 \mathrm{mg} / 24 \mathrm{~h}$ ), ist die Möglichkeit der sicheren Erkennung pathologischer Ergebnisse ausreichend hoch. Vor allem aber ist die DC-Methode als Screeningtest zur Selektion der Urine für die quantitative Bestimmung und zur Absicherung der Ergebnisse der quantitativen Bestimmung geeignet.

\section{Literatur}

1. Pisano, J. J., Crout, J. R. \& Abraham, D. (1962), Clin. Chim. Acta 7, 285-291.

2. Badella, M., Routh, M. W., Gump, B. H. \& Gigliotti, H. J. (1976), Clin. Chem. 22, 2046-2049.

3. Dittmann, J. \& Liem, G. (1966), diese Z. 4, 265.

4. Kraffczyk, F. \& Helger, R. 7. Internat. Congr. Clinical Chemistry, Genf, 12.9.1969.

5. Maruna, H., Maruna, R. F. L. \& Hof, H. M. (1975), Z. Anal. Chem. 274, 26.

6. Wisser, H. \& Knoll, E. (1973), diese Z. 11, 3-14.

Prof. Dr. Rudolf Knuppen, Abteilung für Biochemische Endokrinologie der Medizinischen Hochschule Lübeck, Ratzeburger Allee.160, 2400 Lübeck . . 